\title{
DETERMINAÇÃO SIMULTÂNEA DE RESÍDUOS DE CLORANFENICOL, TIANFENICOL E FLORFENICOL EM LEITE BOVINO POR CROMATOGRAFIA ELETROCINÉTICA MICELAR
}

\author{
Leonardo Pezza* \\ Instituto de Química, Universidade Estadual Paulista, CP 355, 14801-970 Araraquara - SP, Brasil \\ Àngel Ríos \\ Departamento de Química Analítica e Tecnologia dos Alimentos Universidade de Castilla-La Mancha, \\ 13004 Ciudad Real, Espanha \\ Leonor Nozal, Lourdes Arce e Miguel Valcárcel \\ Departamento de Química Analítica, Campus de Rabanales, Universidade de Córdoba, E-14071 Córdoba, Espanha
}

Recebido em 20/4/05; aceito em 22/11/05; publicado na web em 14/6/06

\begin{abstract}
SIMULTANEOUS DETERMINATION OF CHLOROAMPHENICOL, THIAMPHENICOL AND FLORFENICOL RESIDUES IN BOVINE MILK BY MICELLAR ELECTROKINETIC CHROMATOGRAPHY. A micellar electrokinetic chromatographic method (MEKC) is described for determining residues of amphenicols(chloramphenicol,thiamphenicol and florfenicol) in bovine milk. MEKC is conducted by using a separation buffer consisting of $20 \mathrm{mM} \mathrm{Na}_{2} \mathrm{HPO}_{4}, 10 \mathrm{mM} \mathrm{Na}_{2} \mathrm{~B}_{4} \mathrm{O}_{7}, 50 \mathrm{mM}$ SDS at pH 8.0; UV detection at $210 \mathrm{~nm}$ and $10 \mathrm{kV}$ of voltage. The limit of detection ranged from 4.3-5.3 $\mu \mathrm{g} \mathrm{L}^{-1}$. The MEKC method was applied for the simultaneous determination of amphenicols in milk samples spiked with amphenicols at three concentration levels: 10, 30 and $50 \mu \mathrm{g} \mathrm{L}^{-1}$. Recoveries ranging from $91-105 \%$ were obtained by following a simple extraction/preconcentration procedure.
\end{abstract}

Keywords: micelar electrokinetic chromatography; amphenicols; milk.

\section{INTRODUÇÃO}

O uso difundido de antibióticos pelos produtores e médicos veterinários no tratamento de doenças infecciosas de vacas leiteiras, principalmente nas mastites, e a utilização de drogas na alimentação animal, como suplemento de dietas, têm contribuído para a presença de antimicrobianos no leite bovino $^{1-3}$. Outras condições que podem determinar a presença de resíduos de antimicrobianos no leite são a higienização de equipamentos e utensílios da indústria e/ou a adição proposital de drogas para encobrir a deficiência na qualidade higiênica do leite e aumentar seu tempo de vida útil ${ }^{4}$.

A presença de antibióticos no leite, resulta em grande preocupação para a indústria de laticínios, além de representar um risco para a saúde do consumidor.

Em relação ao consumidor, o leite contendo antibióticos pode provocar reações alérgicas, assim como choque anafilático em indivíduos sensíveis. Outro risco a ser considerado é o consumo de leite com altos níveis de resíduos de antibióticos por gestantes, tendo em vista que alguns antibióticos, como nitrofuranos e tetraciclinas, possuem efeitos carcinogênicos que podem levar a alterações no desenvolvimento ósseo fetal. Além de reações alérgicas e de indução de quadros patológicos (ex.: cloranfenicol pode causar anemia crônica), os resíduos de antibióticos possibilitam o risco de indução bacteriana e, posteriormente, a transferência de multirresistência entre os microrganismos através de plasmídios ${ }^{5,6}$. Do ponto de vista da indústria de laticínios, considera-se indesejável a presença de resíduos de antibióticos, em função da sua interferência no crescimento dos cultivos iniciadores durante a elaboração de queijos e iogurtes, causando sérios prejuízos econômicos ${ }^{7}$.

O cloranfenicol é um antibiótico da classe dos anfenicóis, de amplo espectro e altamente efetivo, que foi isolado originalmente

*e-mail: pezza@iq.unesp.br em Streptomyces venezuelae, em $1948^{8}$. Sua ampla utilização em todo mundo tem variado, devido à associação de anemia aplásica (uma doença geralmente fatal) com a exposição ao cloranfenicol, ainda que presente em baixos níveis de concentração $0^{9-11}$. O uso de cloranfenicol em animais produtores de alimentos está banido nos Estados Unidos, na União Européia e em outros países. Recentemente, resíduos de cloranfenicol foram encontrados em carcaças de peixes e em mel produzidos na Ásia ${ }^{12}$.

O tianfenicol e florfenicol são antibióticos com estruturas similares ao cloranfenicol, os quais não estão associados com o aparecimento de anemia aplásica, sendo usados via oral para controle de infecções em seres humanos. Esses antibióticos também são extensivamente utilizados no tratamento de doenças infecciosas de bovinos, suínos e aves.

Embora nenhum desses anfenicóis seja aprovado para uso em gado leiteiro, existe a possibilidade que um ou mais possam ser utilizados para tratamento de uma variedade de doenças que acometem o gado leiteiro, gerando resíduos desses antibióticos no leite.

Pelos fatos acima expostos e visando a proteção do consumidor, a União Européia (EU) e o "Food and Drug Administration" (FDA) dos Estados Unidos estabeleceram um limite máximo de resíduo (MRL) permitido (ou reconhecido como aceitável) para resíduos de antibióticos em alimentos. A Tabela 1 mostra os valores de MRL para os antibióticos pertencentes a classe dos anfenicóis.

A grande maioria dos métodos descritos na literatura para determinação de resíduos de anfenicóis em leite, ovos, tecidos de peixes e de animais produtores de alimentos são métodos cromatográficos $^{10-21}$. Todavia, apenas três deles permitem a determinação simultânea de cloranfenicol, tianfenicol e florfenicol em amostras reais, tais como músculos de peixes ${ }^{15,19}$ e de animais ${ }^{19}$ e leite bovi$\mathrm{no}^{20}$.

Nos últimos anos, a eletroforese capilar (CE) tornou-se uma técnica popular devido à simplicidade, ao alto poder de separação, 
Tabela 1. Limites máximos de resíduos (MRL) de anfenicóis permitidos (ou reconhecidos como aceitáveis) no leite e em outros tecidos animais na União Européia (EU) e nos Estados Unidos (EUA)

\begin{tabular}{lclc}
\hline Anfenicol & Espécies & MRL $(\mu \mathrm{g} / \mathrm{kg})$ & Tecido \\
\hline Cloranfenicol & - & $0(\mathrm{EU}$ e EUA $)$ & - \\
Tianfenicol & Bovina & $50(\mathrm{EU})^{\mathrm{a}}$ & Leite \\
& & $50(\mathrm{EU})^{\mathrm{a}}$ & Músculo \\
& & $50(\mathrm{EU})^{\mathrm{a}}$ & Rim \\
& & $50(\mathrm{EU})^{\mathrm{a}}$ & Fígado \\
& Frango & $50(\mathrm{EU})^{\mathrm{a}}$ & Músculo \\
& & $50(\mathrm{EU})^{\mathrm{a}}$ & Rim \\
Florfenicol & Bovina & $10(\mathrm{EUA})^{\mathrm{b}}$ & Fígado \\
& & $48^{\mathrm{c}}$ & Leite \\
& & $100(\mathrm{EU})^{\mathrm{d}}$ & Leite \\
& Frango & $200(\mathrm{EU})^{\mathrm{d}}$ & Músculo \\
& & $2500(\mathrm{EU})^{\mathrm{d}}$ & Pele \\
& & $750(\mathrm{EU})^{\mathrm{d}}$ & Rígado \\
\hline
\end{tabular}

${ }^{\mathrm{a} O f f i c i a l ~ J o u r n a l ~ o f ~ t h e ~ E u r o p e a n ~ C o m m u n i t i e s ~-~ C o m m i s s i o n ~ R e g u-~}$ lation (EC) No 1000/98 of 13 May 1998, L 142/20; 'FDA Compliance Program Guidance Manual, National Drug Residue Milk Monitoring Program, Food and Drug Administration, Rockville, MD, 1997, Section 7303.039; 'R.E. Baynes et al., Regulatory Toxicology and Pharmacology 29 (1999) 287 e diario Oficial de las Comunidades Europeas - Reglamento (CE) No 2385/1999 de la Comisión de 10 de novembre de 1999, L 288116.

baixo tempo de análise e pequeno consumo de amostra e de solventes ${ }^{22,23}$. Dentre os diferentes modos de CE a cromatografia eletrocinética micelar (MEKC) ${ }^{24,25}$, que faz uso de soluções micelares de tensoativos iônicos, tem se mostrado uma técnica muito atrativa para separação de diversas classes de medicamentos, incluindo antibióticos, agentes antiinflamatórios não esteroidais, esteroidais e analgésicos ${ }^{26}$. Entretanto, a eletroforese capilar com detecção UV-Visível não tem sido aplicada para análise de resíduos de medicamentos em níveis de pequenas partes por bilhão $\left(\mu \mathrm{g} \mathrm{L}^{-1}\right)$, devido à sua menor detectabilidade intrínseca ${ }^{27}$.

No melhor do nosso conhecimento, não existe nenhum método descrito na literatura científica indexada para determinação de resíduos de anfenicóis em leite bovino por CE. Em vista disso, e dada a importância de se monitorar a quantidade de antibióticos da classe dos anfenicóis tanto para a saúde pública, como para as indústrias laticinistas, desenvolveu-se nesse trabalho um método que permite a separação e a quantificação simultânea de anfenicóis (cloranfenicol, tianfenicol e florfenicol) em leite bovino, utilizando a técnica de eletroforese capilar em seu modo MEKC.

Foi obtida suficiente detectabilidade para a determinação de resíduos de anfenicóis em leite bovino através de um procedimento simples de tratamento das amostras, que permitiu a extração e a pré-concentração dos referidos analitos.

Os limites de detecção obtidos neste trabalho foram suficientemente baixos para determinar concentrações de tianfenicol e florfenicol abaixo dos MRL permitidos em leite bovino.

\section{PARTE EXPERIMENTAL}

\section{Reagentes e soluções}

Todas as soluções utilizadas neste trabalho (soluções padrão, soluções estoque, soluções tampão) foram preparadas utilizando água ultrapura obtida de um sistema de purificação de água Millipore milli-Q plus (Bedford, MA, EUA). Os anfenicóis (cloranfenicol, tianfenicol e florfenicol) foram de procedência Sigma (St. Louis, MO, EUA). Soluções padrão estoque (100 $\left.\mu \mathrm{g} \mathrm{mL}^{-1}\right)$ de cada anfenicol foram preparadas em água, sendo armazenadas em frascos escuros e sob refrigeração para evitar possível decomposição. Soluções padrão de trabalho de cada anfenicol (soluções padrão individuais) ou de uma mistura de anfenicóis (soluções padrão combinadas) foram preparadas diaria ou semanalmente, por diluição adequada das correspondentes soluções padrão estoque com água. Padrões preparados em água foram empregados nos estudos envolvendo a otimização das condições de separação e quantificação simultânea dos anfenicóis por CE em seu modo MEKC.

Todos os solventes e reagentes utilizados foram de grau analítico. Metanol, acetonitrila, acetato de etila foram de procedência Merck (Darmstadt,Germany). Os reagentes $\mathrm{NaH}_{2} \mathrm{PO}_{4}, \mathrm{Na}_{2} \mathrm{~B}_{4} \mathrm{O}_{7}$, SDS (Sigma, St. Louis, MO, EUA), $\mathrm{NaOH}$ e $\mathrm{H}_{3} \mathrm{PO}_{4}$ (Merck, Darmstadt, Germany) foram usados na preparação das soluções tampão. Essas soluções foram filtradas através de unidades MillexHV de 0,45 $\mu \mathrm{m}$ da Millipore (Bedford, MA, EUA).

\section{Equipamentos}

Todas as investigações foram realizadas em um sistema de eletroforese capilar ${ }^{3 \mathrm{D}} \mathrm{HP}$ (Hewlett-Packard, Waldbronn, Alemanha) equipado com um autoamostrador e um detector diode array. Um software comercial (ChemStation, HP) foi usado para controlar o sistema, coletar e manipular os dados.

\section{Condições eletroforéticas}

A eletroforese capilar em seu modo MEKC foi realizada em um capilar de sílica fundida (HP, G1600-60311) de $75 \mu \mathrm{m}$ de d.i., $48,5 \mathrm{~cm}$ de comprimento total e $40 \mathrm{~cm}$ de comprimento efetivo. A separação foi conduzida aplicando-se um potencial de $10 \mathrm{kV}$ (polaridade: do ânodo ao cátodo) à temperatura de $25^{\circ} \mathrm{C}$. O tampão de separação empregado foi $\mathrm{Na}_{2} \mathrm{HPO}_{4}(20 \mathrm{mM}), \mathrm{Na}_{2} \mathrm{~B}_{4} \mathrm{O}_{7}(10 \mathrm{mM})$ e SDS $(50 \mathrm{mM})$, cujo $\mathrm{pH}$ foi ajustado ao valor 8,0 com uma solução de $\mathrm{H}_{3} \mathrm{PO}_{4} 1 \mathrm{M}$. Antes de ser utilizado o capilar foi lavado sucessivamente com $\mathrm{H}_{2} \mathrm{O}, \mathrm{HCl} 1 \mathrm{M}, \mathrm{H}_{2} \mathrm{O}, \mathrm{NaOH} 0,1 \mathrm{M} \mathrm{e} \mathrm{H}_{2} \mathrm{O}$ por 5 min cada, e finalmente lavado com o tampão de separação por $15 \mathrm{~min}$. Antes de cada análise o capilar foi condicionado via o seguinte ciclo de lavagem: $\mathrm{H}_{2} \mathrm{O}$ por $5 \mathrm{~min}, \mathrm{NaOH} 0,1 \mathrm{M}$ por $5 \mathrm{~min}, \mathrm{H}_{2} \mathrm{O}$ por $5 \mathrm{~min}$ e tampão de separação por 5 min. $O$ protocolo de injeção foi iniciado com 2 min de $\mathrm{H}_{2} \mathrm{O}, 2$ min do tampão de separação, seguido da injeção hidrodinâmica de padrões e amostras a 20 mbar por $7 \mathrm{~s}$. As medidas fotométricas foram realizadas em um comprimento de onda de $210 \mathrm{~nm}$.

\section{Amostras}

Para a aplicação analítica do método, utilizaram-se 6 amostras recentes de diferentes tipos de leite bovino integral, de origem conhecida e livres de antibióticos. Essas amostras foram fortificadas com soluções padrão de anfenicóis (cloranfenicol, tianfenicol e florfenicol) a três níveis de concentração: 10,30 e $50 \mu \mathrm{g} \mathrm{L}^{-1}$.

\section{Procedimento para a determinação de anfenicóis em amostras de leite bovino integral}

Amostras de leite fortificadas com anfenicóis $(20 \mathrm{~mL})$ foram transferidas para tubos de centrífuga. Na sequiência, foram adicionados $30 \mathrm{~mL}$ de acetato de etila e a mistura foi agitada mecanica- 
mente por $3 \mathrm{~min}$. Após centrifugação a $4000 \mathrm{rpm}$ por $5 \mathrm{~min}$ a fase orgânica foi separada, transferida para frasco apropriado e evaporada até a secura em um evaporador rotatório com banho de água mantido a $45^{\circ} \mathrm{C}$. O resíduo seco foi reconstituído em $300 \mu \mathrm{L}$ de água desionizada, centrifugado por $3 \mathrm{~min}$ a $4000 \mathrm{rpm}$ e injetado diretamente no sistema de eletroforese capilar ou armazenado a $25{ }^{\circ} \mathrm{C}$ até o momento da análise. Para cada tipo de leite analisado uma amostra não fortificada foi tratada segundo o procedimento acima descrito, para um controle negativo.

\section{RESULTADOS E DISCUSSÃO}

A eletroforese capilar com detecção UV/Visível não apresenta detectabilidade suficiente para a quantificação de resíduos de anfenicóis presentes em leite bovino nos níveis de MRL exigidos pela UE e pelo FDA. Para contornar esse problema e aumentar a detectabilidade, um procedimento simples de tratamento das amostras visando o isolamento do analito da amostra biológica ("cleanup") e sua pré-concentração foi desenvolvido. As otimizações das variáveis químicas e instrumentais, bem como as etapas de tratamento da amostra para encontrar as condições mais adequadas do método e sua consequiente aplicação à determinação de resíduos de antibióticos da classe dos anfenicóis em leite bovino, são discutidas a seguir.

\section{Variáveis químicas e instrumentais do método}

Os métodos descritos na literatura que utilizam CE para determinação de anfenicóis em plasma humano ${ }^{26,28}$ e em colírios medicinais $^{29}$ empregam diversos tampões aquosos, tais como ácido acético/acetato de sódio, fosfato, tetraborato de sódio em presença de dodecil sulfato de sódio e acetonitrila. Entretanto, esses métodos só foram aplicados para determinação de um único antibiótico da classe dos anfenicóis. Assim, como primeiro objetivo, procurou-se encontrar um tampão aquoso que permitisse a separação e quantificação simultânea dos anfenicóis estudados (cloranfenicol, tianfenicol e florfenicol).

Testaram-se 4 sistemas tampão: 78\% $\mathrm{NH}_{4} \mathrm{Ac}(10 \mathrm{mM}), \mathrm{pH} 4,6$ e acetonitrila 22\%; $\mathrm{NaH}_{2} \mathrm{PO}_{4}(25 \mathrm{mM}), \mathrm{pH} 2,45 ; \mathrm{Na}_{2} \mathrm{~B}_{4} \mathrm{O}_{7}(25 \mathrm{mM})$, $\mathrm{pH} 9,24 \mathrm{e} \mathrm{Na}_{2} \mathrm{HPO}_{4}(20 \mathrm{mM}), \mathrm{Na}_{2} \mathrm{~B}_{4} \mathrm{O}_{7}(10 \mathrm{mM})$, SDS $(50 \mathrm{mM})$, $\mathrm{pH} \mathrm{9,3.}$

Os resultados obtidos mostraram que a melhor separação dos anfenicóis foi obtida com um sistema tampão constituído por uma mistura de $\mathrm{Na}_{2} \mathrm{HPO}_{4}(20 \mathrm{mM}), \mathrm{Na}_{2} \mathrm{~B}_{4} \mathrm{O}_{7}(10 \mathrm{mM})$, SDS $(50 \mathrm{mM})$, pH 9,3. Escolhido o melhor tampão de separação, a influência do pH foi estudada no intervalo de 6,0 a 10,0, ajustado com uma solução de ácido fosfórico $1 \mathrm{M}$ ou com uma solução de $\mathrm{NH}_{4} \mathrm{OH} 1 \mathrm{M}$. Obtiveram-se os melhores resultados em termos de resolução, sensibilidade e tempo de análise com o sistema tampão acima descrito em $\mathrm{pH}$ 8,0 .

Um estudo da concentração ótima de cada componente do sistema tampão em questão foi realizado em pH 8,0, mantendo-se constante a concentração dos outros componentes. A concentração de $\mathrm{Na}_{2} \mathrm{HPO}_{4}$ foi variada de $10-30 \mathrm{mM}$, a concentração de $\mathrm{Na}_{2} \mathrm{~B}_{4} \mathrm{O}_{7}$ de $5-20 \mathrm{mM}$ e a concentração de SDS de $25-75 \mathrm{mM}$. Os melhores resultados em termos de resolução, sensibilidade e tempo de análise foram obtidos com o seguinte sistema tampão: $\mathrm{Na}_{2} \mathrm{HPO}_{4}$ (20 mM), $\mathrm{Na}_{2} \mathrm{~B}_{4} \mathrm{O}_{7}(10 \mathrm{mM})$, SDS $(50 \mathrm{mM}), \mathrm{pH} 8,0$. Portanto, esse sistema tampão foi utilizado como tampão de separação no presente trabalho.

A adição de modificadores orgânicos, tais como acetonitrila e metanol, também foi estudada. Observou-se que a adição de tais modificadores orgânicos em concentrações de 5 - 10\% (V/V) au- mentou o tempo de análise, sem afetar a resolução e, assim, o seu uso foi descartado.

$\mathrm{O}$ efeito da temperatura na seletividade foi investigado entre 15 e $25^{\circ} \mathrm{C}$. A temperatura de $25{ }^{\circ} \mathrm{C}$ mostrou um melhor compromisso entre seletividade e tempo de corrida dos eletroferogramas.

A influência da diferença de potencial (ddp) aplicada na separação dos anfenicóis foi estudada no intervalo de $5-25 \mathrm{kV}$, observando-se uma diminuição do tempo de análise com o aumento da ddp aplicada. Entretanto, a eficiência de separação dos anfenicóis diminui com o emprego de ddp elevadas. Em vista disso, selecionou-se uma diferença de potencial de $10 \mathrm{kV}$ como voltagem de trabalho.

O melhor valor de comprimento de onda $(\lambda)$ para a detecção dos analitos estudados, determinado com um detector por arranjo de diodos, foi de $210 \mathrm{~nm}$.

A injeção dos analitos (volume de injeção: $25,0 \mathrm{~nL}$ ) foi efetuada em modo hidrostático a 20 mbar, sendo o tempo de injeção hidrostática fixado em $7 \mathrm{~s}$, já que tempos maiores provocaram o aparecimento de picos mal definidos.

Nos estudos acima descritos foram empregados padrões de anfenicóis preparados em água. Com as otimizações acima estabelecidas e utilizando injeção hidrodinâmica, foi possível alcançar limites de detecção (determinado como a concentração do analito que produziu um sinal três vezes maior que o ruído do sistema) em torno de $0,30 \mathrm{mg} \mathrm{L}^{-1}$, sem pré-concentração dos analitos. Observa-se claramente pelos dados da Tabela 1 que a detectabilidade acima obtida não é suficiente para permitir a análise de resíduos de anfenicóis em leite bovino por CE. A fim de aumentar a detectabilidade do método e determinar os anfenicóis em baixas concentrações, desenvolveu-se neste trabalho um procedimento simples de tratamento das amostras que permitiu a extração e a préconcentração dos analitos.

\section{Etapas de tratamento das amostras}

Amostras biológicas são caracterizadas por sua complexidade. Elas contém muitos componentes que podem interferir e tornar sua análise mais difícil. Em vista disso, é necessário um pré-tratamento dessas amostras (extração, pré-concentração, etc.) para que possam ser obtidas em um meio apropriado e em concentrações adequadas para sua injeção no sistema de CE.

Os métodos analíticos descritos na literatura para análise de antibióticos apresentam várias etapas em comum. Um ponto concordante é que uma das primeiras e mais difíceis etapas exigidas para análise de antibióticos é sua extração e o isolamento do analito (“clean-up") da amostra biológica ${ }^{30-34}$. Este é um pré-requisito para todas as metodologias analíticas, embora o grau em que é feito varie amplamente.

As técnicas comumente utilizadas para extração e "clean-up" de antibióticos de amostras biológicas (soro, plasma, urina, leite, etc.) envolvem alguma forma de extração líquido-líquido ou extração em fase sólida. A extração por fluido supercrítico tem atraído interesse devido ao seu potencial para análise de antibióticos ${ }^{35}$, mas devido à exigência de equipamento especializado não tem encontrado ampla aplicação.

A maioria dos métodos existentes para a determinação de resíduos de antibióticos em leite bovino exigem a precipitação das proteínas do leite e a extração em solventes orgânicos polares. Moats ${ }^{36}$ analisou criticamente os vários métodos usados para extração e/ou desproteinização de antibióticos no leite bovino e em tecidos animais.

Os antibióticos da classe dos anfenicóis podem ser extraídos de matrizes biológicas com um solvente orgânico ${ }^{3}$. A desproteini- 
zação/extração geralmente é realizada com metanol, acetonitrila ou acetato de etila ${ }^{31}$

No presente trabalho, todos esses solventes foram testados e o que propiciou os melhores resultados foi acetato de etila. Conforme observado por outros autores ${ }^{3,37,38}$, constatou-se também no presente trabalho que uma única etapa de extração com acetato de etila é suficiente para a extração quantitativa dos antibióticos da classe dos anfenicóis. Além disso, obtiveram-se extratos extremamente límpidos, sem resíduos de gorduras. Portanto, a conjunção dos resultados experimentais acima descritos possibilitou o desenvolvimento de um procedimento simples de desproteinização/extração com acetato de etila, permitindo após etapa de centrifugação e evaporação (para a pré-concentração dos analitos) uma injeção direta dos analitos no sistema de eletroforese capilar.

Os parâmetros eletroforéticos da análise e detalhes gerais do tratamento da amostra para análise de resíduos de anfenicóis (cloranfenicol, tianfenicol e florfenicol) em leite bovino são mostrados na Tabela 2.

Tabela 2. Parâmetros eletroforéticos e tratamento da amostra para análise de resíduos de anfenicóis em leite bovino

\begin{tabular}{|c|c|}
\hline Parâmetros & Método Proposto \\
\hline $\begin{array}{l}\text { Comprimento de } \\
\text { onda de detecção }\end{array}$ & $210 \mathrm{~nm}$ \\
\hline Tampão de separação & $\begin{array}{l}\mathrm{Na}_{2} \mathrm{HPO}_{4}(20 \mathrm{mM}), \mathrm{Na}_{2} \mathrm{~B}_{4} \mathrm{O}_{7}(10 \mathrm{mM}), \\
\mathrm{SDS}(50 \mathrm{mM}), \mathrm{pH} 8,0\end{array}$ \\
\hline Capilar utilizado & $\begin{array}{l}\text { Sílica fundida, } 75 \mu \mathrm{m} \text { de d.i., } 48,5 \mathrm{~cm} \\
\text { de comprimento total e } 40 \mathrm{~cm} \text { de } \\
\text { comprimento efetivo }\end{array}$ \\
\hline Modo de injeção & Hidrodinâmico: a 20 mbar por $7 \mathrm{~s}$ \\
\hline Volume de injeção & $25 \mathrm{~nL}$ \\
\hline $\begin{array}{l}\text { Diferença de } \\
\text { potencial aplicada }\end{array}$ & $10 \mathrm{kV}$ \\
\hline Polaridade & Do ânodo ao cátodo \\
\hline Temperatura & $25^{\circ} \mathrm{C}$ \\
\hline $\begin{array}{l}\text { Tratamento da amostra } \\
\text { (leite bovino) }\end{array}$ & $\begin{array}{l}\text { Precipitação das proteínas e extração } \\
\text { quantitativa dos anfenicóis com acetato } \\
\text { de etila. } \\
\text { Evaporação do solvente em evaporador } \\
\text { rotatório e reconstituição do resíduo em } \\
300 \mu \mathrm{L} \text { de água desionizada }\end{array}$ \\
\hline
\end{tabular}

\section{Validação do método analítico proposto}

Nos métodos bioanalíticos, ao contrário de muitos outros métodos analíticos, as soluções padrão para calibração devem ser preparadas na mesma matriz biológica das amostras (livre do analito) e extraídas da matriz segundo o mesmo procedimento empregado para as amostras ${ }^{39,40}$.

As curvas analíticas para os anfenicóis selecionados (cloranfenicol, tianfenicol e florfenicol) foram obtidas pela fortificação da matriz (leite bovino integral livre de antibióticos) com soluções padrão combinadas de anfenicóis, utilizando-se gráficos de padronização externa e foram lineares em um intervalo de concentração de $9-65 \mu \mathrm{g} \mathrm{L}^{-1}$. As soluções padrão preparadas no leite foram tratadas segundo o mesmo procedimento de extração/pré-concentração descrito para as amostras antes de serem injetadas no equipamento de CE.

Empregou-se o método da padronização externa já que para propósitos quantitativos não se observou nenhuma vantagem significativa usando o método do padrão interno. Na preparação das curvas analíticas foram empregadas seis soluções padrão combinadas, de concentração variável. Cada ponto da reta de calibração de um determinado anfenicol corresponde ao valor médio obtido a partir de três medidas independentes $(n=3)$ da área de pico dos eletroferogramas. Os valores das áreas de pico normalizadas versus as concentrações foram utilizados para aplicar a regressão linear via método dos mínimos quadrados.

O limite de detecção (LOD) $)^{41-43}$ foi considerado como a concentração do analito que produziu um sinal três vezes maior que o ruído do sistema e variou de 4,3 a 5,3 $\mathrm{g} \mathrm{L} \mathrm{L}^{-1}$. O limite de quantificação (LOQ) ${ }^{41-43}$, adotado como a menor concentração do analito que produziu um sinal dez vezes maior que o ruído do sistema com um grau de confiança aceitável (desvio padrão relativo, RSD < 15\%), variou de 8,3 a $9,0 \mu \mathrm{g} \mathrm{L}^{-1}$. A precisão do método proposto (incluindo as etapas de extração/pré-concentração das amostras e a separação por MEKC), expressa como RSD, foi determinada em condições de repetibilidade (intradia) pela análise sucessiva de dez replicatas $(n=10)$ de uma solução padrão combinada preparada na matriz e contendo $20 \mu \mathrm{g} \mathrm{L}^{-1}$ de cada anfenicol. Em todos os casos o valor de RSD foi menor que $6,0 \%$ para a quantificação dos anfenicóis. Uma boa precisão também foi obtida nos tempos de migração (menor que 0,8\% em todos os casos, o que demonstra a eficiência do tampão proposto).

Na Tabela 3 são mostradas as características de desempenho investigadas no processo de validação do método analítico proposto, as quais foram determinadas com o devido rigor estatístico ${ }^{44,45}$ e segundo as diretrizes preconizadas nos procedimentos de validação de métodos cromatográficos e eletroforéticos ${ }^{46,47}$.

A Figura 1 ilustra um eletroferograma típico de uma solução padrão combinada contendo $3 \mathrm{mg} \mathrm{L}^{-1}$ de cada anfenicol em $\mathrm{H}_{2} \mathrm{O}$ e um eletroferograma para uma amostra de leite bovino fortificada com $10 \mu \mathrm{g} \mathrm{L}^{-1}$ de cada anfenicol (após sofrer o processo de extração e pré-concentração proposto). Os picos dos eletroferogramas dessas amostras foram identificados pela comparação com uma base de espectros previamente compilada pelos autores.

A Tabela 4 mostra os valores das recuperações obtidas através da adição de três níveis de concentração de anfenicóis (10, 30 e 50 $\left.\mu \mathrm{g} \mathrm{L}^{-1}\right)$ a um conjunto de seis amostras reais livres de antibióticos. Como pode ser visto, as recuperações variaram de 91 a 105\% (para cinco determinações independentes, $n=5$ ) e o desvio padrão relati-
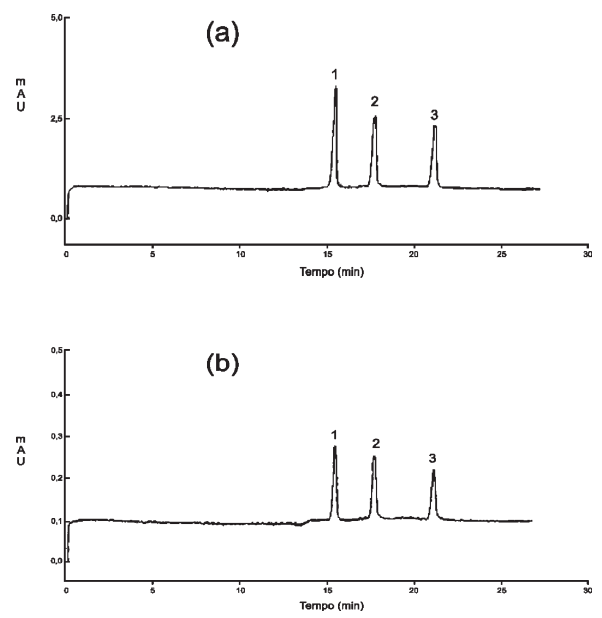

Figura 1. Eletroferogramas obtidos analisando: (a) uma solução padrão combinada contendo $3 \mathrm{mg} \mathrm{L}^{-1}$ de cada anfenicol em meio aquoso, (b) uma amostra de leite bovino fortificada com $10 \mu \mathrm{g} \mathrm{L}^{-1}$ de cada anfenicol após sofrer o tratamento de extração e pré-concentração proposto. 1. tianfenicol; 2. florfenicol; 3. cloranfenicol 
Tabela 3. Características de desempenho do método eletroforético proposto

\begin{tabular}{|c|c|c|c|c|c|c|}
\hline Analito & $y=a+b x^{a}$ & $\mathrm{r}^{\mathrm{b}}$ & $\mathrm{RSD}^{\mathrm{c}}$ Área $(\%)^{\mathrm{c}}$ & $\mathrm{RSD}^{\mathrm{c}}$ tempo $(\%)$ & $\operatorname{LOD}^{\mathrm{d}}\left(\mu \mathrm{g} \mathrm{L}^{-1}\right)$ & $\mathrm{LOQ}^{\mathrm{e}}\left(\mu \mathrm{g} \mathrm{L}^{-1}\right)$ \\
\hline Tianfenicol & $\begin{array}{l}\mathrm{a}=0,006( \pm 0,003) \\
\mathrm{b}=0,463( \pm 0,005)\end{array}$ & 0,9997 & 3,2 & 0,65 & 4,3 & 8,3 \\
\hline Florfenicol & $\begin{array}{l}\mathrm{a}=0,011( \pm 0,004) \\
\mathrm{b}=0,336( \pm 0,009)\end{array}$ & 0,9995 & 4,7 & 0,78 & 4,8 & 8,6 \\
\hline Cloranfenicol & $\begin{array}{l}\mathrm{a}=0,005( \pm 0,004) \\
\mathrm{b}=0,244( \pm 0,007)\end{array}$ & 0,9993 & 5,8 & 0,56 & 5,3 & 9,0 \\
\hline
\end{tabular}

$\overline{\mathrm{a}} \mathrm{a}=$ intercepto; $\mathrm{b}=$ coeficiente angular. Valores obtidos a partir de 3 medidas independentes $(\mathrm{n}=3) ;{ }^{\mathrm{b}} \mathrm{r}=$ coeficiente de correlação; ${ }^{\mathrm{R}} \mathrm{RSD}=$ Desvio padrão relativo das áreas de pico e dos tempos de migração $(n=10)$; ${ }^{\mathrm{L}} \mathrm{LOD}=$ limite de detecção e ${ }^{\mathrm{e}} \mathrm{LOQ}=$ limite de quantificação.

Tabela 4. Análise de amostras de leite bovino fortificadas com diferentes concentrações de antibióticos da classe dos anfenicóis

\begin{tabular}{|c|c|c|c|c|c|}
\hline Amostras ${ }^{a}$ & Anfenicol & $\begin{array}{c}\text { Adicionado } \\
\left(\mu \mathrm{g} \mathrm{L} \mathrm{L}^{-1}\right)\end{array}$ & $\begin{array}{l}\text { Encontrado }^{b} \\
\left(\mu g \mathrm{~L}^{-1}\right)\end{array}$ & $\begin{array}{l}\text { Recuperação média } \\
(\%),(\mathrm{n}=5)\end{array}$ & $\begin{array}{c}\operatorname{RSD}^{\mathrm{c}}(\%) \\
(\mathrm{n}=5)\end{array}$ \\
\hline \multirow[t]{3}{*}{ A } & Cloranfenicol & 10 & 9,1 & 91 & 7,5 \\
\hline & Florfenicol & 10 & 9,3 & 93 & 6,8 \\
\hline & Tianfenicol & 10 & 9,5 & 95 & 6,3 \\
\hline \multirow[t]{3}{*}{ B } & Cloranfenicol & 30 & 28,5 & 95 & 6,9 \\
\hline & Florfenicol & 30 & 29,1 & 97 & 6,6 \\
\hline & Tianfenicol & 30 & 30,9 & 103 & 5,9 \\
\hline \multirow[t]{3}{*}{$\mathrm{C}$} & Cloranfenicol & 30 & 28,8 & 96 & 6,5 \\
\hline & Florfenicol & 30 & 30,6 & 102 & 5,4 \\
\hline & Tianfenicol & 30 & 30,3 & 101 & 6,1 \\
\hline \multirow[t]{3}{*}{$\mathrm{D}$} & Cloranfenicol & 50 & 49,0 & 98 & 6,1 \\
\hline & Florfenicol & 50 & 51,5 & 103 & 6,3 \\
\hline & Tianfenicol & 50 & 50,5 & 101 & 5,8 \\
\hline \multirow[t]{3}{*}{$\mathrm{E}$} & Cloranfenicol & 10 & 9,5 & 95 & 6,3 \\
\hline & Florfenicol & 10 & 10,5 & 105 & 7,3 \\
\hline & Tianfenicol & 10 & 10,2 & 102 & 6,4 \\
\hline \multirow[t]{3}{*}{$\mathrm{F}$} & Cloranfenicol & 50 & 49,0 & 98 & 7,1 \\
\hline & Florfenicol & 50 & 51,0 & 102 & 5,7 \\
\hline & Tianfenicol & 50 & 50,5 & 101 & 5,9 \\
\hline
\end{tabular}

${ }^{\mathrm{a} A m o s t r a s ~ d e ~ l e i t e ~ b o v i n o ~ i n t e g r a l ~ c o n h e c i d a s ~ e ~ l i v r e s ~ d e ~ a n t i b i o ́ t i c o s ; ~}{ }^{\mathrm{b}}$ valores determinados por padronização externa e ${ }^{\mathrm{c} d e s v i o}$ padrão relativo (RSD).

vo (RSD) variou de 5,4 a 7,5\%, comprovando a eficiência do método proposto.

\section{CONCLUSÕES}

Foi desenvolvido um método alternativo utilizando a técnica de eletroforese capilar em seu modo MEKC para determinação quantitativa simultânea de resíduos de anfenicóis em leite bovino. O procedimento analítico proposto neste trabalho é simples, rápido, preciso, exato e suficientemente sensível para permitir o monitoramento de resíduos de tianfenicol e florfenicol abaixo dos valores de MRL estabelecidos pela EU ou pelo FDA (EUA) para leite bovino.

\section{AGRADECIMENTOS}

Ao Ministerio de Ciência e Tecnología de España pelo suporte financeiro por meio de auxílios à pesquisa. L. Pezza agradece à FAPESP, CAPES e CNPq pelo suporte financeiro por meio de auxílios à pesquisa, bolsa de estudo (pós-doutorado) e de produtividade em pesquisa, respectivamente.

\section{REFERÊNCIAS}

1. Sulivan, H. B.; Shaddad, S. A.; Shommein, A. M.; Abdelraraheim, A.; Ibrahim, M. E. M.; Bull. Anim. Helth Prod. Afr. 1990, 38, 65.
2. Bishop, J. R.; White, C. H.; J. Food Protect. 1984, 47, 647.

3. Shenck, F. J.; Callery, P. S.; J. Chromatogr., A 1998, 812, 99.

4. Gustafson, R. H. Em Agricultural Uses of Antibiotics (ACS Symposium Series); Moats, W. A., ed.; American Chemical Society: Washington, DC, 1986, cap. 7.

5. Brady, M. S.; Katz, S. E.; J. AOAC Int. 1992, 75, 738.

6. Brady, M. S.; White, N.; Katz, S. E.; J. Food Protect. 1993, 56, 229.

7. Varnam, A. H.; Sutherland, J. P.; Leche y produtos lácteos: Tecnologia, química y microbiologia, Editorial Acribia: Madrid, 1994.

8. Ehrlich, J.; Smith, R. M.; Penner, M. A.; US pat. 2, 483, 8921949.

9. Settepani, J. A.; J. Am. Vet. Med. Assoc. 1984, 184, 930.

10. Allen, E. H.; J. Assoc. Off. Anal. Chem. 1985, 68, 990.

11. Page, S. W.; Aust. Vet. J. 1991, 68, 1.

12. Quintana-Rizzo, J.; Salter, R.; Saul, S.; Apiacta 2003, 38, 218.

13. Nagata, T.; Saeki, M.; J. Chromatogr. 1991, 565, 471.

14. Nagata, T.; Saeki, M.; J. Liq. Chromatogr. 1993, 16, 2653.

15. Nagata, T.; Oka, H.; J. Agric. Food Chem. 1996, 44, 1280.

16. Munns, R. K.; Holland, D. C.; Plakas, S. M.; J. AOAC Int. 1994, 77, 596.

17. Kijac, J. P.; J. AOAC Int. 1994, 77, 34.

18. Ishii, R.; Tokumaru, Y.; Nose, N.; Shokuhin Eiseigaku Zasshi 1994, 35, 173.

19. Nagata, T.; Saeki, M.; J. Liq. Chromatogr. 1992, 15, 2045.

20. Pfenning, A. P.; Madson, M. R.; Roybal, J. E.; Turnipseed, S. B.; Gonzales, S. A.; Hurlbut, J. A.; Salmon, E. D.; J. AOAC Int. 1998, 81, 714.

21. Penny, L.; Smith, A.; Coates, B.; Wijewickreme, A.; J. AOAC Int. 2005, 88,645 .

22. Tavares, M. F. M.; Quim. Nova 1996, 19, 173.

23. Tavares, M. F. M.; Jager, A. V.; da Silva, C. L.; Moraes, E. P.; Pereira, E. A.; de Lima, E. C.; Fonseca, F. N.; Tonin, F. G.; Micke, G. A.; Santos, M. R.; de Oliveira, M. A. L.; de Moraes, M. L. L.; van Kampen, M. H.; Fujiya, N. M.; J. Braz. Chem. Soc. 2003, 14, 281. 
24. Terabe, S.; Otsuka, K.; Ando, T.; Anal.Chem. 1989, 61, 251

25. Hishi, H.; Terabe, S.; J. Chromatogr., A 1996, 735, 3.

26. Song, J. Z.; Wu, X. U.; Sum, Z. P.; Tian, S. J.; Wang, M. L.; Wang, R. L.; J. Chromatogr., B: Anal. Technol. Biomed. Life Sci. 1997, 692, 445.

27. Simonet, B. M.; Ríos, A.; Valcárcel, M.; Trends Anal. Chem. 2003, 22, 605

28. Jin, W.; Ye, X.; Yu, D.; Song, Q.; J. Chromatogr., B: Anal. Technol. Biomed. Life Sci. 2000, 741, 155.

29. Wang, A.; Zhang, L.; Fang, Y.; Anal. Chim. Acta 1999, 394, 309.

30. Poole, S. K.; Dean, T. A.; Oudsema, J. W.; Poole, C. F.; Anal. Chim. Acto 1990, 236, 3.

31. Barker, S. A.; Long, A. R.; J. Liq. Chromatogr. 1992, 15, 2071.

32. Scheurer, J.; Moore, C. M.; J. Anal. Toxicol. 1992, 16, 264.

33. Petitjean, O.; Tod, M.; Louchahi, K.; J. Pharm. Biomed. Anal. 1995, 13, 817.

34. Fedeniuk, R. W.; Shand, P. J.; J. Chromatogr., A 1998, 812, 3.

35. Vanda, V.; Mikesova, M.; Vejrosta, J.; J. Chromatogr., A 1996, 733, 35.

36. Moats, W. A.; J. Assoc. Off. Anal. Chem. 1990, 73, 343.

37. Wal, J. M.; Peleran, J. C.; Bories, G.; J. Assoc. Off. Anal. Chem. 1980, 63, 1044.
38. Agüí, L.; Guzmán, A.; Yáñez-Sedeño, P.; Pingarrón, J. M.; Anal. Chim. Acta 2002, 461, 65.

39. Causon, R.; J. Chromatogr., B: Anal. Technol. Biomed. Life Sci. 1997, 689 175.

40. Guidance for Industry Bioanalytical Method Validation, FDA, May 2001.

41. Barrón, D.; Jiménez-Lozano, E.; Bailac, S.; Barbosa, J.; J. Chromatogr., A 2002, 767, 313.

42. Fabre, H.; Analusis 1999, 27, 155 .

43. Swartz, M. E.; Krull, I. S.; Analytical Method Development and Validation, Marcel Dekker: New York, 1997.

44. Miller, J. N.; Miller, J. C.; Estadística y Quimiometría para Química Analítica, 4a ed., Prentice Hall: Madrid, 2002.

45. Meier, P. C.; Zund, R. E.; Statistical Methods in Analytical Chemistry, $2^{\text {nd }}$ ed., Wiley: New York, 2000.

46. Ribani, M.; Botolli, C. B. G.; Collins, C. H.; Jardim, I. C. S. F.; Melo, L. F. C.; Quim. Nova 2004, 27, 771.

47. Lanças, F. M.; Validação de Métodos Cromatográficos de Análise, RiMa: São Carlos, 2004. 